\title{
Effect of patient solicitation on mortality among patients receiving hemodialysis in Korea
}

This article was published in the following Dove Press journal:

Patient Preference and Adherence

\author{
Young-Ki Lee' \\ Hyung-Yun Choi ${ }^{2}$ \\ Kiwon $\mathrm{Kim}^{3}$ \\ Ajin Cho' \\ Woo Hun Kang ${ }^{4}$ \\ Young II Choi ${ }^{5}$ \\ Dae Joong Kim ${ }^{6}$
}

'Department of Internal Medicine, Hallym Kidney Institute, Hallym University College of Medicine, Seoul, Korea; ${ }^{2}$ Department of Management of Chronic Disease, Korea Centers for Disease Control \& Prevention, Cheongju, Chungcheongbuk-do, Korea;

${ }^{3}$ Department of Internal Medicine, National Cancer Center, Goyang, Gyeonggi-do, Korea; ${ }^{4}$ Department of Internal Medicine, Samsung Myungin Internal Medicine Clinic, Icheon, Gyeonggi-do, Korea; ${ }^{5}$ Department of Internal Medicine, Young IL Choi Internal Medicine Clinic, Suwon, Gyeonggi-do, Korea; ${ }^{6}$ Department of Internal Medicine, Samsung Medical Center, Sungkyunkwan University School of Medicine, Seoul, Korea

Correspondence: Dae Joong Kim Department of Internal Medicine, Samsung Medical Center, Sungkyunkwan University School of Medicine, 8I, Irwon-ro, Gangnam-gu, Seoul 0635I, Korea

Tel +82 23410344

Fax +82 28295309

Email kimsun4586@naver.com
Background: There is concern that overcompetition and illegal activities such as patient solicitation by some dialysis units may threaten patients' health in Korea. Therefore, we investigated the effect of nephrologists' patient-soliciting activity on hemodialysis practices and patients' survival using the Korean Health Insurance Review and Assessment Service database.

Methods: We selected 19 soliciting hemodialysis facilities and matched them with 19 nonsoliciting facilities located nearby to eliminate location bias. Soliciting behavior was defined as the reduction of medical fees or providing money to attract dialysis patients.

Results: A total of 2,231 incident dialysis patients were included and followed for a median of 37.2 months. Soliciting facilities had a lower percentage of nephrologists, a higher average daily number of hemodialysis patients per physician, and a higher number of hemodialysis patients per nurse compared with non-soliciting facilities. Survival analysis showed that the crude mortality was significantly higher in patients treated in soliciting facilities than in those treated in non-soliciting facilities, even after adjustment for the effects of many other independently predictive covariates.

Conclusions: This study demonstrated that in Korea, the overall mortality rate in incident dialysis patients was higher in those attending soliciting facilities than in those attending non-soliciting facilities.

Keywords: solicitation, hemodialysis, survival

\section{Introduction}

The incidence and prevalence of end-stage renal disease (ESRD) are increasing every year worldwide. According to the Korean Health Insurance Review and Assessment Service database 2013, the cost of care of patients with ESRD is US $\$ 1.4$ billion (32.2\% increase from 2009) and about 69,000 patients received hemodialysis (HD) treatment (22.7\% increase from 2009). ${ }^{1}$ In 2013 , the total number of HD machines was 19,527 and this number has increased at a greater rate than the number of ESRD patients. The average number of HD patients per machine decreased from 3.2 in 2001 to 2.6 in $2012 .^{2}$ An excessive supply of HD machines relative to the number of patients is likely to be a factor in overcompetition between HD facilities.

A conflict of interest exists if there is a clash between a physician's personal financial gain and the welfare of his or her patients. ${ }^{3}$ Although a conflict of interest generally occurs for all physicians who practice fee-for-service medicine, there is a potentially greater conflict of interest for physicians who share in the ownership of for-profit dialysis units in which they treat patients. ${ }^{3}$ The vast majority of Korean 
HD facilities are for-profit. The Korean healthcare system has tended to support resource-intensive treatments because the fee-for-service remuneration system has rewarded their utilization. The number of HD patients attending a dialysis unit is directly related to its profitability. The Korean healthcare system pays for most HD patients' costs and it is possible to charge $90 \%$ of medical expenses to health insurance. Therefore, dialysis facilities can maintain their incomes without charging the patient's share $(10 \%$ of medical expenses) if they control costs and maintain patient volume. There is a concern that overcompetition and illegal activities such as patient solicitation by some dialysis providers may threaten patients' health. Because soliciting facilities are also more likely to engage in multiple cost-saving practices, it is very likely that patient solicitation will be associated with poorer patient outcomes.

Several studies have examined the association between the ownership status of dialysis facilities and patient outcomes. $^{4-7}$ A longitudinal cohort study using the United States Renal Data System (USRDS) demonstrated that patients treated in for-profit dialysis units had a 20\% higher mortality rate and a $26 \%$ lower referral rate for renal transplantation than patients treated in not-for-profit units. ${ }^{4}$ A systematic review and meta-analysis found that HD care in for-profit centers was associated with a higher risk of mortality compared with care in not-for-profit centers. ${ }^{7}$

The hypothesis of our study is that patient-soliciting behavior of the dialysis provider also leads to a decline in the quality of care and may eventually affect the mortality of dialysis patients. However, to date, there has been no study comparing the outcomes of soliciting and nonsoliciting dialysis facilities. Therefore, we investigated the effect of soliciting behavior by dialysis facilities on clinical practice and patients' survival using large-scale Korean registry data, and evaluated the relationship between mortality and various baseline covariates.

\section{Methods}

\section{Data source and study population}

We conducted a cohort study using the Korean Health Insurance Review and Assessment Service database. We identified 20 soliciting dialysis facilities and matched them with 20 nearby non-soliciting facilities to rule out location bias. In order to match the size of each dialysis center, we only included primary healthcare providers while excluding hospitals. Although it is difficult to clearly define patient solicitation, we defined soliciting behavior of HD facilities as the reduction of medical fees or providing money to induce dialysis patients to transfer to the dialysis unit in which their nephrologist had a financial interest. Verification of the solicitation of the dialysis providers was carried out in the same manner as described by "The Korean Doctor's Weekly". ${ }^{8}$ In the guise of a guardian of an HD patient, we asked each dialysis facility by phone whether they could reduce the patient's medical expenses. However, one of the suspected soliciting facilities replied that it did not reduce medical fees, and one non-soliciting facility answered that it could help depending on the patient's situation. Therefore, these two facilities were excluded; the remaining 19 soliciting dialysis facilities and 19 non-soliciting dialysis facilities were included in the final analysis. This study was approved by the Institutional Review Board of Hallym University Kangnam Sacred Heart Hospital, and was conducted according to the Declaration of Helsinki.

Data on the clinical practice of HD of dialysis facilities were collected by the Health Insurance Review and Assessment Service. The analysis was conducted based mainly on the disclosed data on the hemodialysis adequacy evaluation in 2009 and 2010.9,10

To analyze the differences in mortality between soliciting and non-soliciting HD facilities, we initially identified all of the dialysis patients who had commenced HD therapy between 1 January 2009 and 31 December 2012. To use only reliable data, we excluded those patients who survived for less than 90 days, because mortality occurring in the first 90 days was considered to be affected more by preexisting comorbidities or treatment than by the soliciting behavior per se. Those patients receiving HD treatment at more than two different dialysis facilities but over $50 \%$ of their treatment in one facility were included. Patients younger than 18 years of age were excluded.

The comorbidities of the participants were identified by reviewing their medical history during the year prior to the initiation of HD treatment. The list of analyzed comorbidities was determined according to the 5th Korean Classification of Diseases (KCD-5), which is based on the 10th Revision of the International Classification of Diseases (ICD-10). ${ }^{11,12}$ From HIRA database, we collected data such as a unique de-identified number for each patient, age, sex, type of insurance (National Health Insurance versus Medical Aid), list of diagnoses according to the International Classification of Diseases (ICD-10). Written informed consent was waived as the study did not infringe on patient privacy or health status. 


\section{Statistical analysis}

Baseline characteristics of patients attending soliciting and non-soliciting facilities were compared using two-sample for continuous variables and the $\chi^{2}$ test for categorical variables. All patients were followed from the onset of ESRD until death, loss to follow-up, or December 31, 2014 (the end of the observation period). Confirmation of the survival of the study patients was obtained by request to the Ministry of the Interior. Proportional hazards models were used to evaluate the unadjusted and adjusted effects of patient-soliciting behavior on mortality. Statistical analyses were performed using SAS (version 9.2; SAS Institute, Cary, $\mathrm{NC}$ ), and $P$-values $<0.05$ were considered significant.

\section{Results}

\section{Baseline characteristics of the subjects}

A total of 2,231 eligible patients who had started HD treatment between 1 January 2009 and 31 December 2012 were analyzed. At the initiation of dialysis therapy, the mean age of patients was $58.4 \pm 13.9$ years, $61.7 \%$ of patients were men and $56.1 \%$ had diabetes. The number of incident patients in non-soliciting and soliciting facilities was 1,218 (54.6\%) and 1,013 (45.4\%), respectively.

The baseline characteristics of the patients according to the soliciting behavior of the dialysis providers are shown in Table 1. Patients treated in non-soliciting facilities were younger, more often female, and had national health insurance more frequently than those treated in soliciting facilities. Diabetes mellitus was more common in patients attending soliciting facilities compared with those attending non-soliciting facilities. The proportions of patients with various comorbidities differed in the two facility types. Myocardial infarction, congestive heart failure, cerebrovascular accident, peripheral artery disease, chronic pulmonary disease, and peptic ulcer disease were more common in patients treated in soliciting facilities than in those treated in non-soliciting facilities. Liver disease was more common in patients treated in non-soliciting facilities than in those treated in soliciting facilities.

\section{Clinical practice of HD in non-soliciting facilities and soliciting facilities}

The differences in clinical practice of HD treatment between the two groups were analyzed using the Korean Health Insurance Review and Assessment Service database (Table 2). Most of non-soliciting facilities had nephrologists, but only $8.9 \sim 18.0 \%$ of physicians in soliciting facilities were nephrologists. Soliciting facilities had a higher average number of daily HD sessions per physician compared with nonsoliciting dialysis facilities $(26.8 \sim 28.0$ vs $56.6 \sim 60.1)$. Also, the average number of daily HD sessions per nurse of soliciting facilities was significantly higher than non-soliciting facilities $(4.0 \sim 4.1$ vs 4.8$)$. The analysis revealed that the proportion of nurses with more than two years of experience; $81.2 \sim 85.8 \%$ in nonsoliciting dialysis facilities and 59.7 66.7\% in soliciting facilities, respectively. Soliciting dialysis centers had insufficient emergency equipment compared with nonsoliciting dialysis centers. Soliciting facilities water tended to test dialysis adequacy and monitor vascular access infrequently than non-soliciting facilities, but there was no statistically significant difference.

Table I Baseline characteristics of the participants

\begin{tabular}{|c|c|c|c|}
\hline & Non-soliciting facilities $(n=I, 2 \mid 8)$ & Soliciting facilities $(n=I, 0 \mid 3)$ & $P$-value \\
\hline Age (years) & $56.8 \pm \mid 4.8$ & $60.4 \pm 12.6$ & $<0.001$ \\
\hline$>60$ years of age & $548(45.0 \%)$ & $560(55.3 \%)$ & 0.01 \\
\hline Males & 729 (59.9\%) & $648(63.4 \%)$ & 0.05 \\
\hline National Health Insurance & $\mathrm{I}, 099$ (90.2\%) & $882(87.2 \%)$ & 0.03 \\
\hline Diabetes mellitus & $626(51.4 \%)$ & $625(61.7 \%)$ & $<0.001$ \\
\hline Myocardial infarction & $109(9.0 \%)$ & $138(13.6 \%)$ & $<0.001$ \\
\hline Congestive heart failure & $164(13.5 \%)$ & $219(21.6 \%)$ & $<0.001$ \\
\hline Cerebrovascular accident & $106(8.7 \%)$ & 149 (14.7\%) & $<0.001$ \\
\hline Peripheral artery disease & 67 (5.5\%) & $107(10.6 \%)$ & $<0.001$ \\
\hline Chronic pulmonary disease & 118 (9.7\%) & $253(25.0 \%)$ & $<0.001$ \\
\hline Peptic ulcer disease & $139(11.4 \%)$ & $227(22.4 \%)$ & $<0.001$ \\
\hline Liver disease & 206 (16.9\%) & 120 (11.9\%) & $<0.001$ \\
\hline
\end{tabular}

Note: Age is expressed as the mean \pm standard deviation, and all other data are expressed as the number (\%). 
Table 2 Comparison of clinical practices between non-soliciting facilities and soliciting facilities

\begin{tabular}{|l|l|l|l|l|l|}
\hline \multirow{2}{*}{ (Years) } & \multicolumn{2}{l|}{ Non-soliciting facilities (n= 19) } & \multicolumn{2}{l|}{ Soliciting facilities (n=19) } \\
\cline { 2 - 6 } & $\mathbf{2 0 0 9}$ & $\mathbf{2 0 1 0}$ & $\mathbf{2 0 0 9}$ & $\mathbf{2 0 1 0}$ \\
\hline Nephrologists (\%) & 96.3 & 95.6 & 8.9 & 18.0 & $<0$ \\
Career nurse above 2 years (\%) & 81.2 & 85.8 & 59.7 & 66.7 & 60.1 \\
Daily HD per physician & 26.8 & 28.0 & 56.6 & 4.8 & 0.001 \\
Daily HD per nurse & 4.0 & 4.1 & 4.8 & 53.8 & 0.002 \\
Emergency equipment (\%) & 83.3 & 100 & 40.0 & 89.7 & 0.02 \\
Water quality test (\%) & 85.2 & 87.7 & 71.1 & 90.6 & 0.14 \\
HD adequacy test (\%) & 100 & 99.1 & 89.3 & 9.1 \\
Vascular access monitoring (\%) & 99.6 & 94.6 & 82.4 & 0.15 \\
\hline
\end{tabular}

Abbreviation: HD, hemodialysis.

\section{Effects on mortality of patient solicitation by dialysis facilities}

During the median follow-up period of 37.2 months (range, 3.0-59.8 months), 187 patients treated in non-soliciting facilities $(15.4 \%)$ and 232 patients treated in soliciting facilities $(22.9 \%)$ died, which corresponded to death rates of 53.2 and 74.9 deaths per 1000 patient-years, respectively. The 1, 2, 3, and 4-year survival rates of patients in nonsoliciting facilities were $94.8 \%, 90.1 \%, 87.8 \%$, and $84.6 \%$, respectively, and those of patients in soliciting facilities were $95.2 \%, 88.4 \%, 82.9 \%$, and $77.1 \%$, respectively.

Survival analysis using a log-rank test showed that the mortality rate of patients treated in soliciting facilities was significantly higher than that of patients treated in non- soliciting facilities ( $P=0.001$, Figure 1). In the unadjusted hazards analysis, the crude mortality rate among patients treated in soliciting facilities was higher than that among patients treated in non-soliciting facilities (hazard ratio [HR], 1.40; 95\% confidence interval $[\mathrm{CI}], 1.15-1.69 ; P=0.001$ ) (Table 3 ). This survival advantage for non-soliciting facilities over soliciting facilities was not altered even after adjustment for the effects of age, sex, and many other independently predictive covariates (HR, 1.37; 95\% CI, 1.13-1.67; $P=0.001)$ (Table 3).

\section{Subgroup analyses according to baseline covariates and age}

We divided the entire study population into subgroups according to various baseline covariates to compare the

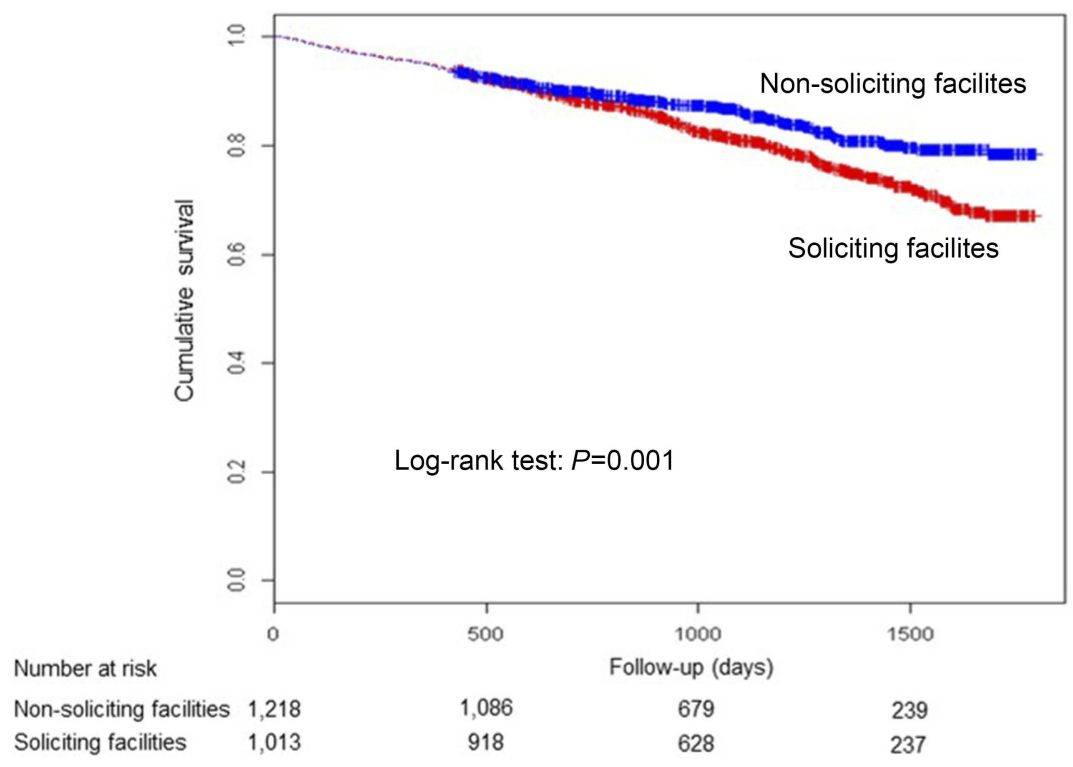

Figure I Crude Kaplan-Meier survival curves for non-soliciting facilities patients and soliciting facilities patients. 
Table 3 Cox proportional hazard model of mortality in dialysis patients

\begin{tabular}{|c|c|c|c|c|}
\hline \multirow[t]{2}{*}{ Variables } & \multicolumn{2}{|l|}{ Unadjusted } & \multicolumn{2}{|l|}{ Adjusted } \\
\hline & Hazard ratio (95\% C.I.) & P-Value & Hazard ratio (95\% C.I.) & $P$-value \\
\hline Soliciting facilities & $1.40(1.15-1.69)$ & 0.001 & $1.37(1.13-1.67)$ & 0.001 \\
\hline Age (per 10 years) & $1.09(1.01-1.17)$ & 0.02 & $1.10(1.02-1.18)$ & 0.011 \\
\hline Sex (Male) & $1.33(1.08-1.63)$ & 0.007 & $1.28(1.05-1.58)$ & 0.017 \\
\hline Diabetes mellitus & $1.30(1.07-1.59)$ & 0.009 & $1.27(1.04-1.56)$ & 0.019 \\
\hline Myocardial infarction & $1.61(1.13-2.29)$ & 0.009 & $1.81(1.27-2.59)$ & 0.001 \\
\hline Congestive heart failure & $1.11(0.86-1.43)$ & $0.4 I$ & - & \\
\hline Peripheral artery disease & $0.79(0.58-1.08)$ & 0.14 & - & \\
\hline Cerebrovascular accident & $1.14(0.85-1.54)$ & 0.39 & - & \\
\hline Chronic pulmonary disease & $1.00(0.78-1.28)$ & 0.99 & - & \\
\hline Peptic ulcer disease & $0.99(0.77-1.26)$ & 0.91 & - & \\
\hline Liver disease & $1.21(0.91-1.6 \mathrm{I})$ & 0.18 & - & \\
\hline
\end{tabular}

mortality within subgroups for non-soliciting and soliciting facilities. Regardless of sex, the mortality rates were consistently higher in patients treated in soliciting facilities compared with those treated in non-soliciting facilities in each subgroup without any comorbidities with the exception of the subgroup of patients with peripheral artery disease. And we also found the mortality rates were consistently higher in patients treated in soliciting facilities compared with those treated in non-soliciting facilities in all subgroup without any comorbidities, including the subgroup of patients with peripheral artery disease (HR, 2.15; 95\% CI, 1.44-3.21, $P<0.001)$ (Figure 2).

Because the age of patients at the start of their dialysis treatment could be a key determinant of mortality, we performed subsequent analyses in which mortality rates were assessed separately for the older age group ( $\geq 60$ years) and the younger age group ( $<60$ years). Figure $\mathrm{S} 1$ shows that there were no significant differences in mortality rates between non-soliciting facilities and soliciting facilities for patients who started dialysis at $\geq 60$ years of age, and with the exception of a subgroup of patients with peripheral vascular disease,

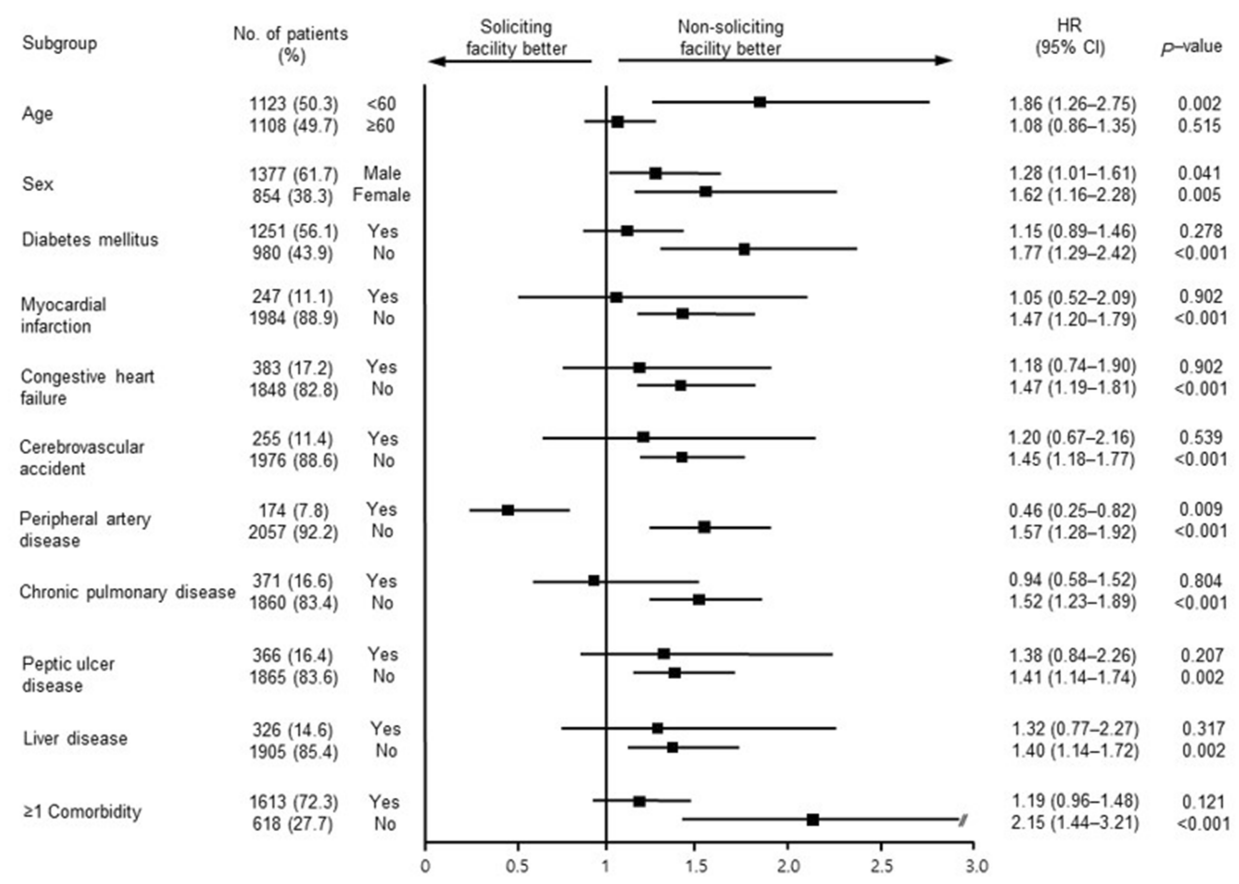

Figure 2 Subgroup analyses comparing hazard ratios for mortality between non-soliciting facilities patients and soliciting facilities patients. 
there were no significant differences in mortality rates between older patients treated in non-soliciting and soliciting facilities, regardless of their comorbidities.

In the younger age group ( $<60$ years), the overall mortality rates were significantly higher in patients treated in soliciting facilities compared with those treated in nonsoliciting facilities (HR, 1.86; 95\% CI, 1.26-2.75; $P=0.002$ ) (Figure $\mathrm{S} 2$ ). There was a significantly higher mortality rate for diabetic patients treated in soliciting facilities than for diabetic patients treated in nonsoliciting facilities (HR, 1.74; 95\% CI, 1.02-2.95; $P=0.041$ ), whereas there was no significant difference in the mortality rates between nondiabetic patients treated in non-soliciting and soliciting facilities. In the younger age subgroups without comorbidities, the mortality rates of patients treated in soliciting facilities were consistently higher than for those treated in non-soliciting facilities.

\section{Discussion}

In this study, we found that patients treated in soliciting facilities had a $30 \%$ higher mortality rate than those treated in non-soliciting facilities. Although previous studies have investigated whether the type of ownership of a dialysis facility affects patient outcomes, ${ }^{4-7}$ no studies have examined the effects of patient solicitation by dialysis facilities on patients' survival. Also common but not new is the practice of nephrologists trying to recruit dialysis patients to their own HD facilities in which they have a financial interest. ${ }^{13}$ Although the number of such incidents is unknown, professional quality-oriented renal organizations have become aware of an increased number of complaints regarding patient solicitation. ${ }^{8,14}$ However, no objective data were available indicating that medical treatments by non-soliciting HD facilities were better than those by soliciting facilities. This study sought to examine whether patient solicitation was associated with the patient mortality rate, accounting for geographic region and patient characteristics.

In the survival analysis, soliciting dialysis facility was an independent risk factor for death in patients starting dialysis, even after accounting for multiple patient characteristics. Our findings suggest that the medical treatment provided for ESRD patients may differ between nonsoliciting and soliciting HD facilities. In particular, the poorer patient survival at soliciting facilities may result from a more aggressive response to incentives to economize compared with that at non-soliciting facilities. Although measures to reduce costs may simply lead to greater efficiency, they may also compromise patient welfare if they entail reducing dialysis time, purchasing cheaper, possibly less effective dialyzers and dialysis machines, and hiring fewer nephrologists and registered nurses. ${ }^{3}$ Also, our analysis showed that soliciting facilities had a lower percentage of nephrologists, a higher average daily number of HD patients per physician, and a higher average daily number of HD patients per nurse compared with non-soliciting dialysis facilities. They also had insufficient emergency equipment and their testing of water quality and dialysis adequacy was inadequate. Therefore, considerably more research is needed to compare the adequacy of medical treatment in non-soliciting and soliciting facilities.

This study also demonstrated the effects on mortality of interactions between patient solicitation by HD facilities and various baseline covariates. Interestingly, we found that the mortality risk of the group treated in soliciting facilities was significantly increased for patients aged $<60$ years old (1.7-fold) and those without comorbidities (2.2-fold).

We present the results of an analysis of populationbased registry data. HD patients in this study had a lower death rate than that noted in the USRDS annual data report. ${ }^{15}$ This difference may be because of the relatively younger age of our subjects and their lower prevalence of preexisting cardiovascular diseases compared with those analyzed in the USRDS. The difference in mortality rate may be associated with our exclusion of patients who survived for less than 90 days. However, the overall death rate of HD patients in our study was similar to that in the previously published Korean registry data for $2014 .^{16}$

This study has some important limitations. Because it was observational, we could not exclude that some of the observed differences between non-soliciting and soliciting facilities related to residual confounding factors, despite our adjustment for numerous characteristics of the patients and facilities. Our study could not indicate causality between patient solicitation by dialysis facilities and mortality because of limitations in the registry data. Data about several other factors that might potentially influence mortality, such as nutrition, inflammation, adequacy of dialysis, CKD-MBD, anemia, other laboratory biomarkers, and medical treatments of HD patients, were unavailable. We also could not identify the causes of death of HD patients. The differences in transplantation rates, hospitalization rates, and medical costs between the two groups were 
not investigated. However, we have presented the results of our analysis of the population-based registry data for HD patients covered by both the National Health Insurance and Medical Aid, our sample size was relatively large and the length of follow-up was comparatively long, and we tried to eliminate local bias by matching soliciting and non-soliciting HD facilities in nearby geographic regions.

It is an illegal action that the reduction of medical fees or providing money to induce patients to transfer to the dialysis unit in which their nephrologist had a financial interest. To reduce the cost of medical treatment to the poor is not soliciting behavior. But, providing money to induce patients to transfer to the dialysis unit is a soliciting behavior According to the data of the "The Korean Doctor's Weekly", 8 the percentage of dialysis facilities which provide reduced amount of coinsurance payment to the patients was $27.2 \%$. In addition, the percentage of dialysis facilities providing free transportation services was $53.4 \%$.

To eradicate the soliciting behaviors of HD facilities, dialysis-specific quality assurance is crucial. Developed countries have their own survey and certification programs to provide monitoring to ensure these facilities continue to meet basic requirements, even after initial certification of dialysis units. In the United States, the ESRD Networks play a pivotal role in quality improvement projects, data collection, and response to patient complaints, and provide quality oversight services to facilities performing chronic dialysis treatment. ${ }^{17}$ In Korea, the accreditation of dialysis units by the Korean Society of Nephrology and the assessment of hemodialysis adequacy by the Korean Health Insurance Review and Assessment Service have now been implemented; however, concerns about conflicts of interest remain. To hold dialysis units accountable for the quality of care they provide, quality indicators should be established to measure the soliciting behavior of individual dialysis centers. Reform is needed of the current reimbursement system for dialysis services, which rewards efforts by the facility to keep costs low and maintain patient volume but does not provide incentives to maximize clinical outcomes. ${ }^{4}$ In addition, standards for the establishment of HD units, ${ }^{18}$ organizations to provide quality oversight services to dialysis facilities, and education of nephrologists about nonsoliciting issues are needed in Korea.

\section{Conclusions}

This study demonstrated that in Korea, patients treated in soliciting HD facilities have higher mortality than those treated in non-soliciting facilities. Because soliciting facilities use less labor and equipment per dialysis treatment to create profit, these cost-saving practices may be associated with poorer prognoses. The reimbursement system for dialysis services must give feedback to providers of care to HD patients. Most importantly, health-care policy makers should consider the finding that treatment of dialysis patients in soliciting facilities is associated with poor outcomes.

\section{Acknowledgments}

This work was supported by a grant from the Korean Medical Devices Industry Association (KMDIA).

\section{Disclosure}

The authors declare that they have no competing interests in this work.

\section{References}

1. Korean Health Insurance Review and Assessment Service. A report of 4rd Hemodialysis adequacy assessment. [cited April 8, 2015]. Available from: www.hira.or.kr. Accessed December 16, 2016.

2. Jin DC, Han JS. Renal replacement therapy in Korea, 2012. Kidney Res Clin Pract. 2014;33:9-18. doi:10.1016/j.krcp.2014.01.002

3. Moss AH. Ethical dilemmas facing nephrology: past, present, and future. In: Skorecki K, Chertow GM, Marsden PA, Taal MW, Yu ASL, editors. Brenner and Rector's the Kidney. 10th ed. Philadelphia, PA: Elsevier; 2016:2558-73.

4. Garg PP, Frick KD, Diener-West M, Powe NR. Effect of the ownership of dialysis facilities on patients' survival and referral for transplantation. $N$ Engl $J$ Med. 1999;341:1653-60. doi:10.1056/ NEJM199911253412205

5. Dalrymple LS, Johansen KL, Romano PS, et al. Comparison of hospitalization rates among for-profit and nonprofit dialysis facilities. Clin J Am Soc Nephrol. 2014;9:73-81. doi:10.2215/ CJN.04200413

6. Brunelli SM, Wilson S, Krishnan M, Nissenson AR. Confounders of mortality and hospitalization rate calculations for profit and nonprofit dialysis facilities: analytic augmentation. BMC Nephrol. 2014;15:121. doi:10.1186/1471-2369-15-121

7. Devereaux PJ, Schünemann HJ, Ravindran N, et al. Comparison of mortality between private for-profit and private not-for-profit hemodialysis centers: a systematic review and meta-analysis. Jama. 2008;288:2449-57.

8. Kim JK. Current status and problems of Illegal-operating hemodialysis units in Korea. Kidney Res Clin Pract. 2012;31(Suppl 2):S99-S103.

9. Korean Health Insurance Review and Assessment Service. A report of 2009 Hemodialysis adequacy assessment. [cited July 2010]. Available from: www.hira.or.kr. Accessed December 16, 2016.

10. Korean Health Insurance Review and Assessment Service. A report of 2010 Hemodialysis adequacy assessment. [cited 2012 February]. Available from: www.hira.or.kr. Accessed December 16, 2016.

11. Charlson ME, Pompei P, Ales KL, MacKenzie CR. A new method of classifying prognostic comorbidity in longitudinal studies: development and validation. J Chronic Dis. 1987;40:373-83. doi:10.1016/ 0021-9681(87)90171-8 
12. Quan H, Sundararajan V, Halfon $\mathrm{P}$, et al. Coding algorithms for defining comorbidities in ICD-9-CM and ICD-10 administrative data. Med Care. 2005;43:1130-9. doi:10.1097/01.mlr.0000182534.19832.83

13. Ozar DT, Kristensen C, Fadem SZ, Blaser R, Singer D, Moss AH. Nephrologists' professional ethics in dialysis practices. Clin J Am Soc Nephrol. 2013;8:840-4. doi:10.2215/CJN.08490812

14. Park SE, Kang DH, Son SH, et al. Current status of hemodialysis units in Korea. Kidney Res Clin Pract. 1999;18:661-71.

15. US Renal Data System. USRDS 2016 Annual Data Report: Atlas of Chronic Kidney Disease and End-Stage Renal Disease in the United States. National Institutes of Health, National Institute of Diabetes and Digestive and Kidney Diseases; 2016.
16. Kim H, Kim KH, Park K, et al. A population-based approach indicates an overall higher patient mortality with peritoneal dialysis compared to hemodialysis in Korea. Kidney Int. 2014;86:991-1000. doi:10.1038/ki.2014.163

17. Wish JB, Meyer KB. ESRD networks: past, present, and challenges for the future. Clin J Am Soc Nephrol. 2012;7:1907-14. doi:10.2215/ CJN.10251011

18. Lee YK, Kim K, Kim DJ. Current status and standards for establishment of hemodialysis units in Korea. Korean J Intern Med. 2013;28:274-84. doi:10.3904/kjim.2013.28.3.274 


\section{Supplementary materials}

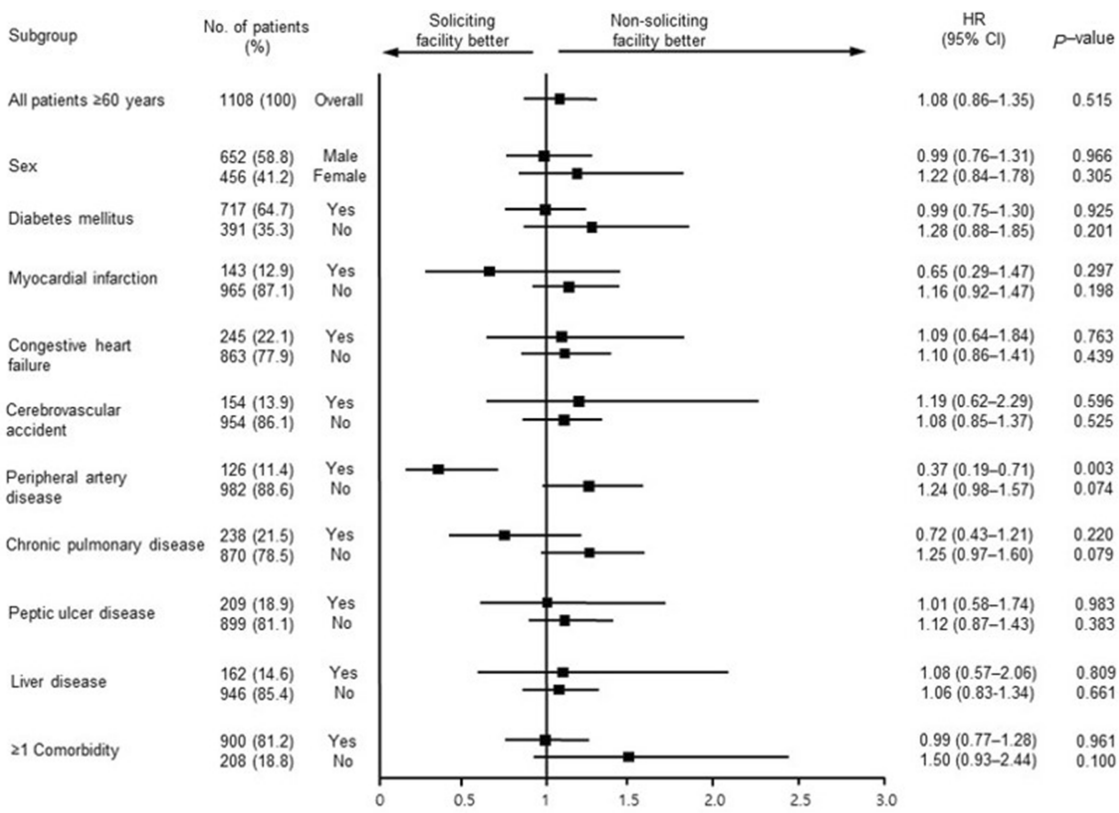

Figure SI Subgroup analyses comparing hazard ratios for mortality between non-soliciting facilities patients and soliciting facilities patients aged more than 60 years.

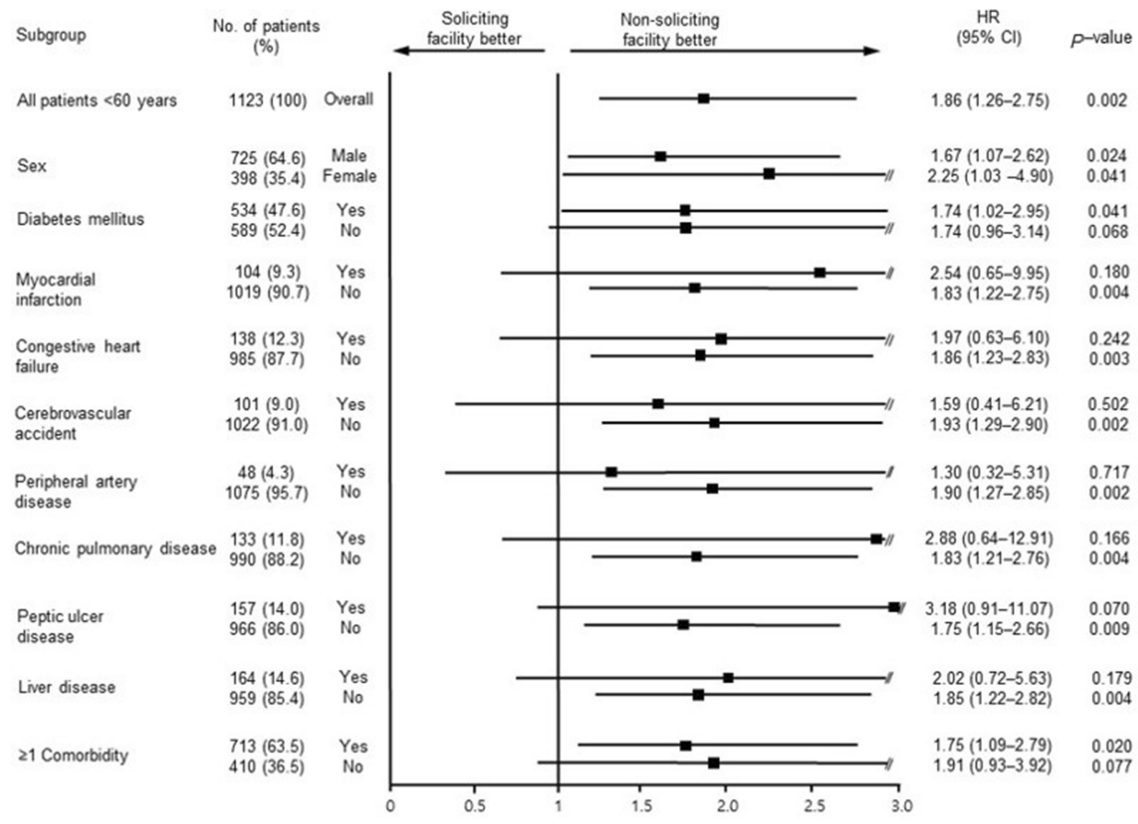

Figure S2 Subgroup analyses comparing hazard ratios for mortality between non-soliciting facilities patients and soliciting facilities patients aged less than 60 years. 


\section{Publish your work in this journal}

Patient Preference and Adherence is an international, peer-reviewed, open access journal that focusing on the growing importance of patient preference and adherence throughout the therapeutic continuum. Patient satisfaction, acceptability, quality of life, compliance, persistence and their role in developing new therapeutic modalities and compounds to optimize clinical outcomes for existing disease states are major areas of interest for the journal. This journal has been accepted for indexing on PubMed Central. The manuscript management system is completely online and includes a very quick and fair peer-review system, which is all easy to use. Visit http:// www.dovepress.com/testimonials.php to read real quotes from published authors. 